\title{
Successfully Treated Hepatitis B Positive Refractory Myasthenic Crisis with Rituximab
}

\author{
R Kumar Singh ${ }^{1}$, Deepika Joshi ${ }^{2}$, Shivani Rath ${ }^{3}$
}

\begin{abstract}
Myasthenia gravis (MG) is an autoimmune disorder of neuromuscular transmission, which presents with fluctuating and variable weakness in ocular, bulbar, limb, and respiratory muscles resulting from an antibody-mediated, T-cell-dependent immunologic attack on the postsynaptic membrane of the neuromuscular junction. Although treatment of MG and myasthenic crisis is based on few specific principles, it is highly individualized. We report a successfully treated case of refractory myasthenic crisis who was on a ventilator for 7 months (210 days), perhaps the longest from India, and required multiple cycles of plasma exchange, intravenous immunoglobulin infusion, and one cycle of rituximab. It exemplifies the role of highly individualized therapy and interdisciplinary cooperation in management of refractory myasthenic crisis.

Keywords: Myasthenia gravis, Myasthenic crises, Rituximab.

Indian Journal of Critical Care Medicine (2020): 10.5005/jp-journals-10071-23320
\end{abstract}

\section{INTRODUCTION}

Myasthenia gravis (MG) is an autoimmune disorder of neuromuscular transmission, which presents with fluctuating and variable weakness in ocular, bulbar, limb, and respiratory muscles resulting from an antibody-mediated, T-cell-dependent immunologic attack on the postsynaptic membrane of the neuromuscular junction. Refractory MG is defined by status of the patient that remains unchanged or worsens after corticosteroids and at least two other immunosuppressive agents, used in adequate doses for an adequate duration, with persistent symptoms or side effects that limit functioning, as defined by the patient and the physician. ${ }^{1}$ Evidence-based international consensus guideline is available for management of $\mathrm{MG}^{2}$ but treatment choices are highly individualized and clinicians face many theoretical and practical difficulties in choosing the right therapy in cases of refractory MG and myasthenic crisis. Myasthenic crisis is defined by worsening of myasthenic weakness requiring intubation or noninvasive ventilation to avoid intubation, except when these measures are employed during routine postoperative management, ${ }^{3}$ and it is managed on same principles employed for refractory MG. Experiences of use of agents, like cyclophosphamide, tacrolimus, and rituximab, with mixed outcomes, limited to case reports and case series, are present. Successful use of rituximab has been reported from India in a limited numbers of patients in few studies ${ }^{4}$ and few case reports ${ }^{5}$ in management of refractory MG. Most studies reported an average 2-3 weeks of intensive care unit (ICU) stay ${ }^{3,4}$ in myasthenic crisis. The longest duration of crises reported till date is 661 days. $^{6}$

We report a challenging case of myasthenic crisis with an ICU stay of 9 months (7 months on ventilator), refractory to rapid immunomodulation therapy where we used rituximab with partial success after failure of several cycles of plasma exchange (PE) and repeated infusions of intravenous immunoglobulin (IVIG). Duration of ventilator support and ICU stay is, perhaps, the longest period reported from India.

\section{Case Description}

A 35-year-old male patient presented in our out-patient department (OPD) in June 2014 with diplopia, ptosis, and excessive
${ }^{1-3}$ Department of Neurology, Institute of Medical Sciences, Banaras Hindu University, Varanasi, Uttar Pradesh, India

Corresponding Author: Deepika Joshi, Department of Neurology, Institute of Medical Sciences, Banaras Hindu University, Varanasi, Uttar Pradesh, India, Phone: +91 9918978666, e-mail: drdeepikajoshi73@ gmail.com

How to cite this article: Singh RK, Joshi D, Rath S. Successfully Treated Hepatitis B Positive Refractory Myasthenic Crisis with Rituximab. Indian J Crit Care Med 2020;24(1):71-72.

Source of support: Nil

Conflict of interest: None

fatigability and was diagnosed with AchR antibody-positive generalized MG. Contrast enhanced computerized tomography (CECT) of the chest revealed an ill-defined heterogeneously enhancing lesion in the anterior mediastinum. He was treated with cholinesterase inhibitors (pyridostigmine) and low dose of oral steroids with success. He was doing well till December 2014 when he developed relapse of symptoms in the form of diplopia, ptosis, and fatigability with additional manifestation of dysarthria and dysphagia. His doses of pyridostigmine were titrated to maximum possible doses and a short tapering course of oral steroids was given with addition of a steroid-sparing drug, azathioprine, which was titrated up to $150 \mathrm{mg}$ in divided doses. Patient slowly improved and underwent thymectomy (diagnosed with hepatitis B virus-positive in preoperative screening) in February 2015. Histopathology was suggestive of thymoma B1 with cystic degeneration. He was doing well on symptomatic treatment, till end of January 2016 when he developed relapse of symptoms with dyspnea following an episode of fever with cough. He was admitted in ICU on February 2016 in crisis and was kept on ventilator after endotracheal intubation. He went for five cycles of PE without much improvement and subsequently IVIG $(2 \mathrm{~g} / \mathrm{kg})$ was infused over 5 days, and he started showing improvement and was weaned off ventilator after 5 weeks in March 2016.

After 24 hours of removal of the ventilator support of 5 weeks, the patient again needed the ventilator support. After 2 weeks of failed attempt of weaning of the ventilator support, IVIG was infused 
again for 5 days without any success. We went for five cycles of PE in May 2016 without any success and four cycles of rituximab (300 $\mathrm{mg} / \mathrm{m}^{2}$ ) were then infused in June 2016 with partial success when he intermittently managed to stay off the ventilator for few hours. After much interdisciplinary deliberation to rule out any possible cause responsible for refractory state crisis, we repeated CECT again to rule out thymoma as well as sent hepatitis B DNA copies. Tenofovir was added after gastroenterologist consultation. The patient remained ventilator dependent on polypharmacy with our constant attempt to titrate symptomatic and immunosuppressive therapy till October 2016 and we faced multiple challenges in the form of ventilatorassociated pneumonia and recurrent leucopenia. Azathioprine could not be titrated more than $150 \mathrm{mg}$ due to leucopenia and Tenofovir was stopped after consideration of its potential to cause leucopenia. The patient again went for 10 cycles of alternate-day PE in October 2016 and slowly he was weaned off the ventilator completely in November 2016 after 7 months on the ventilator. He was transferred out to wards after 9 months of ICU stay. Over the next two months, the patient developed ptosis and diplopia, despite being on maximum tolerable doses of symptomatic and immunosuppressive treatment for which an additional 10 cycles of PE were administered. Finally, his tracheostomy closure was done in January 2018 after a period of 11 months. The patient went for a total of 45 cycles of PE, 2 cycles of IVIG infusion, and 1 cycle (four doses- $300 \mathrm{mg} / \mathrm{m}^{2}$ ) of rituximab during his hospital stay and presently he is doing well on symptomatic and immunosuppressive therapy.

\section{Discussion}

Most of the patients of refractory MG and myasthenic crisis are managed by clinician's judgment and available agents especially after failure of PE and IVIG. Newer agents like cyclophosphamide, tacrolimus, and rituximab are being used infrequently around the world in management of crisis. Rituximab, a monoclonal anti-CD20 antibody, inhibits the progression of B cells through antibodydependent cellular cytotoxicity, ${ }^{7}$ complement-dependent cytotoxicity, and apoptosis ${ }^{8}$ and has an excellent safety profile with no major contraindications. We used this drug with partial success. As our patient stayed for 9 months in ICU and for 7 months on the ventilator, the use of rituximab hastened the weaning off from the ventilator complemented by other immunomodulating treatment.

\section{Conclusion}

Myasthenia gravis is a treatable disorder but requires individualized therapy, interdisciplinary approach, and high level of patience. Any case of refractory MG and myasthenic crisis should be thoroughly evaluated and all available medical therapies should be judiciously tried for a better outcome. Rituximab is a promising agent in the treatment of refractory myasthenic crisis. One should be patient when managing such patients as sometimes the patient may have a prolonged ventilator stay.

\section{References}

1. Jaretzki III A, Barohn RB, Ernstoff RM, Kaminski HJ, Keesey JC, Penn AS, et al. Myasthenia gravis: recommendations for clinical research standards: task force of the medical scientific advisory board of the myasthenia gravis foundation of America. Neurology 2000;55(1): 16-23. DOI: 10.1212/WNL.55.1.16.

2. Sanders DB, Wolfe GI, Benatar M, Evoli A, Gilhus NE, Illa I, et al. International consensus guidance for management of myasthenia gravis: executive summary. Neurology 2016;87(4):419-425. DOI: 10.1212/WNL.0000000000002790.

3. Panda S, Goyal V, Behari M, Singh S, Srivastava T. Myasthenic crisis: a retrospective study. Neurol India 2004;52(4):453-456.

4. Sharma S, Lal V, Prabhakar S, Agarwal R. Clinical profile and outcome of myasthenic crisis in a tertiary care hospital: a prospective study. Ann Indian Acad Neurol 2013;16(2):203-207. DOI: 10.4103/09722327.112466.

5. Rahul LY, Dakua T, Mukherjee A. Refractory myasthenia gravis treated successfully with rituximab: a case report. Ann Indian Acad Neurol 2017;20(4):436-437. DOI: 10.4103/aian.AIAN_233_17.

6. Selecky PA, Ziment I. Prolonged respiratory support for the treatment of intractable myasthenia gravis. Chest 1974;65(2):207-209. DOI: 10.1378/chest.65.2.207.

7. Clynes RA, Towers TL, Presta LG, Ravetch JV. Inhibitory fc receptors modulate in vivo cytotoxicity against tumor targets. Nat Med 2000;6(4):443-446. DOI: 10.1038/74704.

8. Reff ME, Carner K, Chambers KS, Chinn PC, Leonard JE, Raab R, et al. Depletion of $B$ cells in vivo by a chimeric mouse human monoclonal antibody to CD20. Blood 1994;83(2):435-445. DOI: 10.1182/blood. v83.2.435.435. 